\title{
PHARMACEUTICAL BULLETIN
}

Vol. 2 No. 3

September 1954

39. Yo Ueda : Samen Saponin von Thea sinensis L. III*.

Über die Konstitution des Thea-sapogenols. (1).

(Pharmaz. Institut d. Universität Kyushu**)

Vor kurzem* hat der Verfasser aus den Samen von Thea sinensis L. ein Saponin vom Schmp. 224 225 (korr.) isoliert. Nach der Hydrolyse mit Salzsäure und Ätznatron wurde gezeigt, dass die Bestandteile des Saponins die folgende Gleichung befriedigen.

Thea-saponin $+5 \mathrm{H}_{2} \mathrm{O}=$ Angelikasäure + Glucuronsäure + Galaktose + Xylose $\mathrm{C}_{57} \mathrm{H}_{88(86)} \mathrm{O}_{26}$

$$
+ \text { Arabinose + Thea-sapogenol }
$$

Einige Beobachtungen der Konstitution des Thea-sapogenols werden hier berichtet.

In der Fig. 1 und 2 werden die Ultraviolette-1) und Infrarot-Absorptionsspektren ${ }^{2)}$ des Thea-sapogenols und seines Reduktionsprodukts Dihydroteasapogenol gegeben. In der UV-Region hat das Thea-sapogenol sowie sein Reduktionsprodukt ein schwaches und breites Maximum bei $275 \sim 295 \mathrm{~m} \mu$ ( $\log \varepsilon=1.7$ und 1.6), aber beide Minima bei $240 \mathrm{~m} \mu$ sind verschieden in ihren Tiefen. Die IR-Absorptionen der beiden Verbindungen enthalten die (mutmasslichen) charakteristischen Gruppenfrequenzen aus Tabelle I.

\begin{tabular}{|c|c|c|c|c|}
\hline Kurve & $\mathrm{OH}$ & $\mathrm{CO}$ & $\mathrm{OH}$ & $\mathrm{RR}^{\prime} \mathrm{C}=\mathrm{CHR}$ \\
\hline (a) Thea-sapogenol & 2.9 & 5.8 & $9.05, \quad 9.25$ & 11.95 \\
\hline (b) Dihydrotheasapogenol & 2.85 & - & 9.05 & 11.95 \\
\hline
\end{tabular}

Auf Grund der IR-Absorptionsspektren und der Tatsache, dass Thea-sapogenol neutral ist $^{3)}$ und mittels Diazomethan nicht reagiert, wird mindestens die Anwesenheit von einer Carbonyl-gruppe angedeutet. Das Carbonyl war ganz beständig gegen Carbonylreagenzien, aber bei der Behandelung mit Natrium und iso-Amylalkohol. wurde es zu einem Produkt Dihydrotheasapogenol, $\mathrm{C}_{30} \mathrm{H}_{50} \mathrm{O}_{6}$, von Schmp. $326^{\circ}$ (korr.) reduziert. Das letzte zeigte in dem IR-Spektrum (Tabelle I) die charakteristische Frequenz nicht mehr.

Thea-sapogenol sowie Dihydrosapogenol wurden katalytisch kaum reduziert wie bei vielen Amyrin-typus Triterpene der Fall ist, doch färbten sie sich mit Tetranitromethan deutlich gelb, was das Vorhandensein einer Doppelbindung andeutet.

Thea-sapogenol lieferte nur amorphe Acetylderivate beim Behandeln mit Essigsäureanhydrid-Pyridin oder Essigsäureanhydrid-Essigsaures Natrium, aber mit Hilfe von Essigsäureanhydrid-Schwefelsäure wurde ein gut kristallisierendes Acetat vom schmp. 272 $274^{\circ}$ (korr.) erhalten. Ebenso lieferte Dihydrosapogenol ein Acetat vom Schmp. 145 $146^{\circ}$. Diese zwei Acetate unterscheiden sich scharf durch Löslichkeit in Lösungsmitteln.

* Mitteilung I : M. Ishidate, Y. Ueda : J. Pharm. Soc. Japan, 72, 1523 (1952); Mitteilung II: Y. Ueda : Ibid., 72, 1525 (1952).

** Katakasu, Fukuoka (上田 陽).

1) Die Bestimmungen wurden in $80 \%$ äthanolischen Lösungen ausgeführt.

2) Für die Aufnahme danke ich Herrn H. Kamata des technologischen Institutes der Universität Tokyo.

3) Mitteilung II: (loc. cit.). 
Bemerkenswert ist die Tatsache, dass die beiden Acetate ebenso sechs Acetyl-reste aufwiesen und beim Verseifen der beiden Thea-sapogenol und Dihydrosapogenol wieder unverändert erhalten wurden. Diese wäre nur dadurch erklärlich, dass das Carbonyl des Theasapogenol enolisierbar ist und so die sechs Saverstoffe vollständig zur Acetylierung fähig sind, was aber noch weitere Beweise erfordert.

Bei der $\alpha$-Glykol-Bestimmung nach Criegee verbrauchte Thea-sapogenol ein equivalentes Bleitetraacetat, demnach ist die Anwesenheit von ein Paar sekundärer Alkoholgruppe sehr wahrscheinlich.

Das Thea-sapogenol lieferte keine nennenswertige Reaktionsprodukte mit Triphenylmethylchlorid, $p$-Toluolsulfonsäurechlorid oder Phthalsäureanhydrid, und gab nur amorph Additionsprodukte mit Aceton, Cyclohexanon und Benzaldehyd.

Das oxydative Abbauen mit all den üblichen Methoden-Criegee'sche, Oppenauer'sche, Chromsäureanhydrid und Permanganat-oxydationen des Sapogenols sowie seiner Acetonverbindung ergaben nur harzige Produkte, dessen Reinigung ohne Erfolg war. Es erschwerte die weiteren Studien sehr.

Schliesslich um die Grundskelette des Sapogenols zu bestätigen, wurde Thea-sapogenol zur Destillation mit Selen versetzt. Aus dem Reaktionsprodukt wurden 1,2,3,4-Tetramethylbenzol und Sapotalin erhalten.

Aus den oben erhaltenen Resultaten kann man schliessen, dass Thea-sapogenol wahrscheinlich ein neues Triterpen von der Zusammensetzung $\mathrm{C}_{30} \mathrm{H}_{48(46)} \mathrm{O}_{6}$ ist, in dem Moleküle ein Carbonyl, eine Doppelbindung und fünf Hydroxylgruppen mit ein paar ortho-ständigem, enthält.

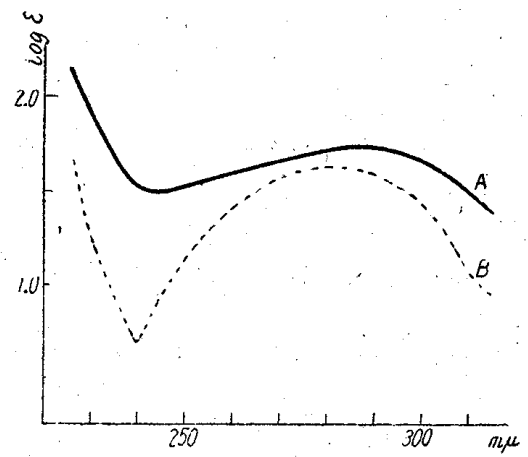

A : Thea-sapogenol

B : Dihydrotheasapogenol

Fig. 1.

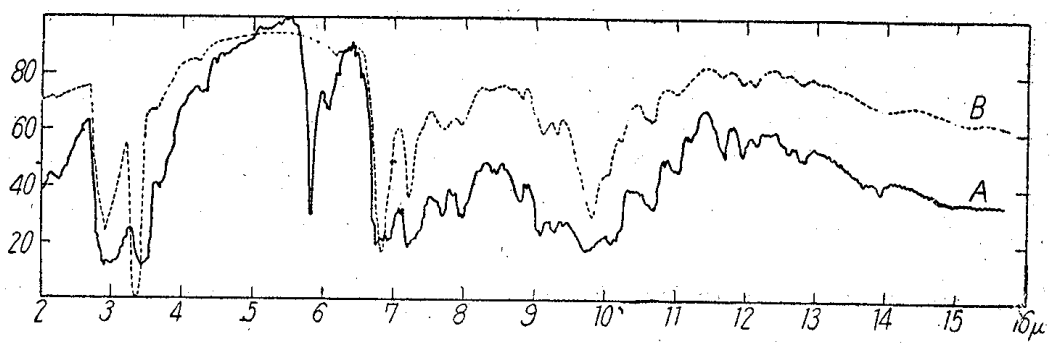

A : Thea-sapogenol (in Nujol)

B : Dihydrotheasapogenol (in Nujol) Fig. 2.

Diese Arbeit wurde unter der Leitung von Herrn Prof. M. Ishidate und Herrn Prof. T. Momose durchgeführt, wofür ich meinen besten Dank ausspreche. Auch danke ich Herrn E. Kimura, Herrn T. Hattori und Frl. T. Kawano für die Ausführung der Elementaleanalyse.

\section{Beschreibung der Versuche}

Behandlung mit Diazomethan und Ätzalkali-Das Thea-sapogenol wurde in Äther suspendiert oder in Alkohol gelöst, mit der ätherischen Lösung des $\mathrm{CH}_{2} \mathrm{~N}_{2}$ zugesetzt und einige Tage lang stehen gelassen. Stickstoff entwickelte sich nicht, und das Thea-sapogenol wurde regeneriert. Zur alkoholischen Lösung des Thea-sapogenols wurde die wässerige Lösung des $\mathrm{NaOH}$ zugesetzt (in der Hitze oder Kälte) und die ausgeschiedenen Kristalle wurden mit wenig Wasser (od. wenig wasserhaltigem Alkohol) gewaschen. Diese Kristalle waren identisch mit dem Thea-sapogenol.

Reduktion mit Na und $i$ so-A mylalkohol (Bildung von Dihydrotheasapogenol)-1 $\mathrm{g}$ Thea-sapogenol wurde in $100 \mathrm{ccm}$ iso-Amylalkohol gelöst und diesem $2 \mathrm{~g} \mathrm{Na}$ zugesetzt. Nach dem Erkalten wurde die Reaktionslösung mit $20 \mathrm{ccm}$ Wasser und $6 \mathrm{ccm}$ AcOH gesauert und im Vakuum von den Lösungsmitteln abdestilliert. Der Rückstand gab bei zweimaligem Umlkristallisieren aus $80 \%$ EtOH farblose Tafeln vom Schmp. 326 327 (korr.). $\mathrm{C}_{30} \mathrm{H}_{50} \mathrm{O}_{6}$-Ber. : C, 71.11; H, 9.95. Gef.: C, 71.09, 71.50, 70.90, 70.87; $\mathrm{H}, 9.61,9.64,9.82,9.75$.

Es sublimierte nicht, färbte sich mit $\mathrm{Ac}_{2} \mathrm{O}-\mathrm{H}_{2} \mathrm{SO}_{4}$ kirschrot-rotviolett-blauviolett. Es färbte sich mit $\mathrm{C}\left(\mathrm{NO}_{2}\right)_{4}$ gelb. 
Bestimmung nach der Ogg, Porter und Willits'schen Methode ${ }^{4}$-Zu etwa $0.1 \mathrm{~g}$ Thea-sapogenol wurden $2 \mathrm{ccm} \mathrm{Ac}_{2} \mathrm{O}$-Pyridin-Mischung (1:3) zugesetzt, mit einem Glasstopfen, welcher mit Pyridin angefeuchtet wurde, gestöpselt und bei $90 \sim 95^{\circ}$ erwärmt. Nach 2 Stunden wurden $20 \mathrm{ccm}$ Wasser zugefügt, 2 Minuten geschüttelt und mit fliessendem Wasser gekühlt. Der Stopfen und die innere Wand des Gefässes wurden mit $1 \mathrm{ccm}$ EtOH (od. BuOH) gewaschen, und mit $0.2 \mathrm{~N}$ äthanolischem $\mathrm{NaOH}$ titriert. Indikator : $0.1 \%$ Kresolrot-0.1\% Thymolblau $(1: 3)$.

Verbindung

Thea-sapogenol

Menthol (sekundäre $\mathrm{OH} \cdots \cdot 1$ )

Erythrodiol (sekundäre $\mathrm{OH} \cdots \cdots 1$, primäre $\mathrm{OH} \cdots 1$ )
Gefundene Zahl der OH-Gruppe

$\begin{array}{llllll}2.64 & 2.60 & 2.97 & 2.31 & 2.25 & 2.28 \\ 0.62 & 0.54 & 0.56 & & & \\ 1.34 & 1.28 & 1.74 & 1.56 & 1.46 & \end{array}$

Acetat des Thea-sapogenols-2 $\mathrm{g}$. Thea-sapogenol wurden in $20 \mathrm{ccm} \mathrm{Ac}_{2} \mathrm{O}$ gelöst und 10 Tropfen konz. $\mathrm{H}_{2} \mathrm{SO}_{4}$ unter Kühlung hinzugefügt und über Nacht stehen gelassen. Danach wurde dieses 30 Minuten bei $70^{\circ}$ erwärmt, in Eiswasser gegossen, abgesaugt und mit Wasser gewaschen. Prismen aus EtOH (od. verd. AcOH) vom Schmp. 272 274 (korx.). $\mathrm{C}_{42} \mathrm{H}_{60} \mathrm{O}_{12}-$ Ber. : C, 66.64; H, 7.99; mol. Gew., 756.9. Gef.: C, 66.10, 66.10, 65.81; H, 8.15, 8.16, 8.13; mol. Gew., 759.6 (Rast). Das Acetat in EtOH färbte sich mit $\mathrm{C}\left(\mathrm{NO}_{2}\right)_{4}$ stark gelb und wurde katalytisch mit Pd-Kohle nicht hydriert.

Acetat des Dihydrotheasapogenol-Die Acetylierung wurde analogerweise aufgearbeitet. Die Reaktionsmischung wurde ins Eiswasser gegossen, und mittels Benzol ausgezogen. Der Auszug wurde mit $\mathrm{Na}_{2} \mathrm{CO}_{3}$-Lösung und Wasser gewaschen, und mit $\mathrm{Na}_{2} \mathrm{SO}_{4}$ getrocknet. Die konzentrierte Benzollösung wurde durch eine Alumina Säule geflossen und mit Benzol eluiert. Hierbei wurden Kristalle erhalten. Nadeln von Schmp. $145 \sim 146^{\circ}$ aus Benzol-Petroläther. $\mathrm{C}_{42} \mathrm{H}_{62} \mathrm{O}_{12}$-Ber. : C, 66.60; H, 8.24. Gef: C, $66.65 ; \mathrm{H}, 7.83$.

Bestimmuug der Acetylgruppe-Etwa $0.03 \mathrm{~g}$ Acetat wurden mit $5 \mathrm{ccm} 2 \mathrm{~N} 75 \%$-äthanolischem $\mathrm{KOH} 2$ Stunden in siedendem Wasserbad verseift. Nach dem Erkalten wurde $1 \mathrm{ccm}$ Phosphorsäure hinzugerügt und mit Wasserdampf destilliert. Das Destillat (etwa $50 \mathrm{ccm}$ ) wurde mit $0.02 \mathrm{~N} \mathrm{NaOH}$ titriert. Indikator: Phenolphtalein.

Zahl der Acetylgruppen

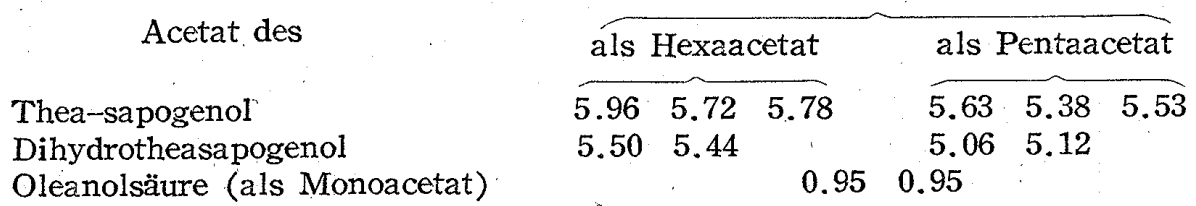

Nach dem Umkristallisieren aus $80 \%$ EtOH stimmte jeder dieser verseiften Substanzen mit Theasapogenol oder Dihydrosapogenol überein.

Bestimmung nach der Criegee'schen Methode-(Die zwei Reaktionsbedingungen A und B werden beschrieben, und B wird in den Klammern gezeigt.) Etwa 0.1 (0.025)g Thea-sapogenol wurden in $30(10) \mathrm{ccm} \mathrm{Ac}_{2} \mathrm{O}$ und $6(4) \mathrm{ccm} \mathrm{Pb}(\mathrm{OAc})_{4}-\mathrm{Ac}_{2} \mathrm{O}$ (bekannter Konzentration) gelöst, und 20 (72) Stunden bei $18 \sim 20^{\circ}$ stehen gelassen. $40(10) \mathrm{ccm}$ Wasser und $6(4) \mathrm{ccm} \mathrm{KJ}-\mathrm{NaOAc}-$ Lösung $(2 \mathrm{~g} \mathrm{KJ}$ und $50 \mathrm{~g}$ $\mathrm{NaOAC}$ in $100 \mathrm{ccm}$ Wasser) wurden hinzugefügt, mit $0.1 \mathrm{~N}-\mathrm{Na}_{2} \mathrm{~S}_{2} \mathrm{O}_{3}$-Lösung titriert.

\begin{tabular}{|c|c|c|c|c|c|c|}
\hline \multirow{2}{*}{ Verbindung } & \multicolumn{5}{|c|}{ Zahl der $\alpha$-Glykolgruppen } & \\
\hline & & A & & & $\mathrm{B}$ & \\
\hline Thea-sapogenol & 0.95 & 1.03 & 1.11 & 1.42 & 1.15 & 1.35 \\
\hline Hederagenin & 0.24 & 0.46 & & 0.31 & 0.28 & 0.28 \\
\hline
\end{tabular}

Dehydrierung- $20 \mathrm{~g}$ rohes Pulver des Thea-sapogenol wurde mit $40 \mathrm{~g}$ Selen gemischt, im Metallbad anfangs bei $350^{\circ}$ und darauf bei $300 \sim 320^{\circ}$ erhitzt. Nach 50 Stunden wurde das Reaktionsgemisch unterbrochen und mit Äther extrahiert. Das ölige Extrakt wurde im Vakuum fraktioniert.

$\begin{array}{lrrlr}\text { 1) } & 9 \mathrm{~mm} & \text { Gelbes Öl } & 2 \mathrm{ccm} \\ \text { 2) } & 9 \mathrm{~mm} & 122 \sim 132^{\circ} & \text { Gelbes Öl } & 1 \mathrm{ccm} \\ \text { 3) } & 9 \mathrm{~mm} & 132 \sim 142^{\circ} & \text { Orange-gelbes Öl } & 1 \mathrm{ccm} \\ \text { 4) } 5 \mathrm{~mm} & \sim 145^{\circ} & \text { Orange-gelbes Öl } & 1 \mathrm{ccm} \\ \text { 5) } 5 \mathrm{~mm} & 145 \sim 155^{\circ} & \text { Oranges Öl } & 1 \mathrm{ccm} \\ \text { 6) } & 2 \mathrm{~mm} & 148 \sim 153^{\circ} & \text { Oranges Öl } & 1 \mathrm{ccm} \\ \text { 7) } & \text { Rückstand } & & \end{array}$

4) Ind. Eng. Chem., Anal. Ed., 17, 394 (1945). Ich gewann 0.5 0.6 OH Zahlen bei Menthol und 1.3 1.4 OH-Zahlen bei dem Erythrodiols. Diese Besimmung ist dann nicht quantitativ, wenn die sekundäre $\mathrm{OH}-$ Gruppe in Frage kommt. 
Die Fraktion 1 wurde wieder fraktioniert.

a) $12 \mathrm{~mm} \quad \sim 75^{\circ} \quad$ einige Tropfen

b) $12 \mathrm{~mm} \quad 75 \sim 100^{\circ} \quad$ einige Tropfen

c) $12 \mathrm{~mm} \quad 100-115^{\circ} \quad$ Gelbes Öl $1 \mathrm{ccm}$

d) $7 \mathrm{~mm} \quad-115^{\circ} \quad$ wenig

Die Fraktion (a) wurde bromiert und aus $\mathrm{MeOH}$ umkristallisiert. Farblose Nadeln vom Schmp. 200 201 . Es war identisch mit Dibrom-1,2,3,4-tetramethylbenzol. $\quad \mathrm{C}_{10} \mathrm{H}_{12} \mathrm{Br}_{2}-\mathrm{Ber} .: \mathrm{C}, 41.13 ; \mathrm{H}$, 4.14. Gef. : C, 41.24; H, 4.03.

Die Fraktion(3)wurde zum Pikrat geführt. Orange-gelbe Nadeln vom Schmp. 128 129 (aus EtOH). Es war mit Sapotalin-pikrat identisch. $\mathrm{O}_{19} \mathrm{H}_{17} \mathrm{O}_{7} \mathrm{~N}_{3}-\mathrm{Ber} .: \mathrm{N}, 10.5$. Gef. : N, 10.71 .

\section{Zusammenfassung}

Thea-sapogenol, dessen provisorische Zusammensetzung als $\mathrm{C}_{30} \mathrm{H}_{48} \sim{ }_{46} \mathrm{O}_{6}$ angesehen wurde, hat in sich mindestens ein Carbonyl, eine $\alpha$-Glykolgruppe und eine schwer hydrierbare Doppelbindung. Da das Sapogenol Hexaacetylderivate (Schmp. 272 274 ${ }^{\circ}$ ) lieferte und das Acetat noch an sich die Färbung mit $\mathrm{C}\left(\mathrm{NO}_{2}\right)_{4}$ intakt behielt, ist das Carbony1 wahrscheinlich enolisierbar. Beim Behandeln mit Natrium und Amylalkohol wurde ein Reduktionsprodukt, Dihydrotheasapogenol (Schmp. 326 327 ), erhalten. Bei der Destillation des Thea-sapogenols mit Hilfe von Selen wurden 1,2,3,4-Tetramethylbenzol und Sapotalin isoliert. So scheint das Sapogenol ein neues Triterpen von Amyrin-typus zu sein.

(Eingegangen am 18. März 1954)

40. Ken'ichi Takeda, Tameto Okanishi, and Ariyoshi Shimaoka: Studies on the Steroidal Components of Domestic Plants. IV ${ }^{1)}$. Constituents of Yucca Species. (1).

(Research Laboratories, Shionogi \& Co., Ltd.*)

Plants of the Yucca species, together with those of Agave and Dioscorea spp., have widely been studied as the material for steroidal sapogenins. Marker and others ${ }^{2,3}$ have isolated 10 kinds of sapogenins from 42 kinds of the Yucca spp. growing in Mexico and the United States, including sarsasapogenin (from 19 plants), smilagenin (from 18), gitogenin (from 5), tigogenin (from 3), yuccagenin (from 3), kammogenin (from 3), and furcogenin, mexogenin, samogenin, and texogenin (from 1 plant each). In general, however, it is known that the quality and the amount of a steroidal sapogenin in a plant differ according to the age of the plant, position, and the season of collection, as well as the habitat ${ }^{4)}$. Examination of the sapogenin contained in the domestic Yucca plants was therefore carried out and the results obtained were markedly different from that reported by Marker, et al.

The Yucca plants used for the present series of experiments are shown in Table I.

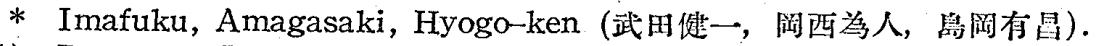

1) Part III : J. Pham. Soc. Japan, 73, 84 (1953).

2) R. E. Marker, et al.: A. D. I. Document 23, 8, 4.

3) R. E. Marker, et al.: J. Am. Chem. Soc., 69, 2167 (1947).

4) R. E. Marker, et al. : J. Am. Chem. Soc., 69, 2221 (1947). 